Article

\title{
Long-Term Monitoring of the Impacts of Disaster on Human Activity Using DMSP/OLS Nighttime Light Data: A Case Study of the 2008 Wenchuan, China Earthquake
}

\author{
Xue Li ${ }^{1}$, Cong Zhan ${ }^{1}$, Jianbing Tao ${ }^{2, *}$ and Liang $\mathrm{Li}^{3}$ \\ 1 Key Laboratory of Earthquake Geodesy, Institute of Seismology, China Earthquake Administration, \\ NO. 40 Hongshance Road, Wuhan 430071, China; leexue1211@126.com (X.L.); zhan_cong@yeah.net (C.Z.) \\ 2 Key Laboratory for Geographical Process Analysis \& Simulation of Hubei Province/School of Urban and \\ Environmental Sciences, Central China Normal University, NO. 152 Luoyu Road, Wuhan 430079, China \\ 3 The Third Academy of Engineering of Surveying and Mapping in Sichuan Province, \\ NO. 2 Xinjun Road in Xindu District, Chengdu 610500, China; liliang1987wuda@163.com \\ * Correspondence: taojb@mail.ccnu.edu.cn; Tel.: +1-387-106-3601
}

Received: 1 March 2018; Accepted: 9 April 2018; Published: 10 April 2018

check for updates

\begin{abstract}
Time series monitoring of earthquake-stricken areas is significant in evaluating post-disaster reconstruction and recovery. The time series of nighttime light (NTL) data collected by the defense meteorological satellite program-operational linescan system (DMSP/OLS) sensors provides a unique and valuable resource to study changes in human activity (HA) because of the long period of available data. In this paper, the DMSP/OLS NTL images' digital number (DN) is used as a proxy for the intensity of HA since there is a high correlation between them. The purpose of this study is to develop a methodology to analyze the changes of intensity and distribution of HA in different areas affected by a 2008 earthquake in Wenchuan, China. In order to compare the trends of HA before and after the earthquake, the DMSP/OLS NTL images from 2003 to 2013 were processed and analyzed. However, their analysis capability is greatly limited owing to a lack of in-flight calibration. To improve the continuity and comparability of DMSP/OLS NTL images, this study developed an automatic intercalibration method to systematically correct NTL data. The results reveal that: (1) compared with the HA before the earthquake, the reconstruction and recovery of the Wenchuan earthquake have led to a significant increase of HA in earthquake-stricken areas within three years after the earthquake; (2) the fluctuation of HA in a severely-affected area is greater than that in a less-affected area; (3) recovery efforts increase development in the most affected areas to levels that exceeded the rates in similar areas which experienced less damage; and (4) areas alongside roads and close to reconstruction projects exhibited increased development in regions with otherwise low human activity.
\end{abstract}

Keywords: Wenchuan earthquake; nighttime light; intercalibration; human activity; reconstruction and recovery

\section{Introduction}

A catastrophic earthquake measuring Mw 7.9 struck Sichuan Province in Southwestern China on 12 May 2008, representing one of the country's worst natural disasters. Approximately 87,150 people were killed or missing and another 374,643 were injured. The Wenchuan Earthquake severely struck 237 counties and cities, seriously damaging over 100,000 square kilometers of area, with the direct economic loss reaching 845.2 billion RMB [1]. The seismic intensity at the epicenter was XI degree; 
more than half of China was affected, and many other Asian countries, such as Thailand, Vietnam, and Pakistan, felt the earthquake's subsequent shock.

Remote sensing technology has played a vital role in accessing the material losses and impacts on human society and environmental landscapes caused by the Wenchuan earthquake [2,3]. The application of remote sensing technology in post-disaster assessment has been widely studied, such as in building damage [4], quantitative seismic damage [5], landslides, and other secondary disasters [6,7]. The impact of disasters on the ecological environment is also a hotspot of research, such as damaged croplands [8], soil mass [9], and ecosystems of natural reserves [10].

Damage from an earthquake occur in a short period of time, but the impacts on humans and society will last far longer: studies have shown that natural disasters, such as large earthquakes, have long-term effects on human society [11,12]. Determining how to use remote sensing technology to carry out long-term, sustained, and effective monitoring of impacts to earthquake-stricken areas is of great significance for disaster recovery policy-making and research on disaster loss prevention and reduction.

Different from daytime remote sensing, nighttime light (NTL) remote sensing provides a unique perspective on human activity (HA). In addition to having wide applications in the estimation of social and economic parameters, such as gross domestic product (GDP), population and electricity, and urbanization monitoring, NTL remote sensing data is increasingly used in assessing the impact of major events, such as social unrest and natural disasters [13]. Li made use of the defense meteorological satellite program-operational linescan system (DMSP/OLS) images and Suomi National Polar-orbiting Partnership (NPP) satellite Visible Infrared Imaging Radiometer Suite (VIIRS) sensor images to evaluate the Syrian civil war (2011 to 2014), finding a high correlation between reductions in NTL intensity and refugee movements [14].

NTL data has played a more and more important role in earthquake damage assessment. Kohiyama assessed the city light loss or reduction after the West India earthquake in 2001 using the DMSP/OLS daily images, determining that the reduction in NTL intensity was in "good agreement" with the field investigations [15]. In the field of recovery assessment research, Koma identified the differences among the reconstructed areas and the reconstructing areas after the Sichuan earthquake using the DMSP/OLS daily images and estimated the pace of reconstruction [16]. Thomas quantified the fluctuation of DMSP/OLS annual images caused by the Sumatra tsunami and recovery, and revealed the significant relationships between the nighttime imagery brightness and per capita expenditures, and spending on energy and food [17].

DMSP/OLS possesses abundant archival data and wide spatial coverage. However, DMSP/OLS has no on-board calibration mechanism. Furthermore, annual NTL data acquired by different satellites have no strict intercalibration. The lack of continuity and comparability means that these data cannot be directly used to extract time series information [18]. Individual composites have been intercalibrated via an empirical procedure. The existing methods of intercalibration often require manual selection of invariant target areas [19-23]; in turn, an automatic intercalibration method needs to satisfy the assumption that the NTL value of most pixels in two period images is stable [24]. However, because of rapid development over the past two decades, it is difficult to find a region in China with stable NTL. Accordingly, this poses a challenge to the existing methods of intercalibration.

This study used DMSP/OLS time series NTL images to assess HA in the Wenchuan earthquake-stricken areas and to compare the changes in intensity and distribution of HA before and after the disaster. The objective of the research is to reveal the continuing impacts of the Wenchuan Earthquake and post-earthquake reconstruction on HA through the long-term remote sensing monitoring. The novelty of our research is that an automatic intercalibration method is proposed to improve the continuity and comparability of DMSP/OLS time series NTL images in the developing regions. The novel calibration method helps to unify the comparative basis of HA, and our research framework provides a reference for the study of the long-term impacts of earthquakes and other types of disasters. 


\section{Materials and Methods}

\subsection{Study Areas}

The seismic intensity distribution of Wenchuan earthquake, which was compiled by a field survey from the China Earthquake Administration, is used to select the study area (Figure 1). In this paper, the effect of Wenchuan earthquake on the Earth's surface is described by the levels of Chinese Seismic Intensity Scale. The relationship between Chinese Seismic Intensity Scale and Modified Mercalli Intensity Scale is shown in Appendix A. The area of the XI level region, which has two separate centers located in Wenchuan County and Beichuan County, respectively, is approximately $2419 \mathrm{~km}^{2}$. The distribution of the Wenchuan XI level region is along the NE-SW direction, forming a "strip" with a $66 \mathrm{~km}$ long axis and a $20 \mathrm{~km}$ short axis. The Beichuan XI level region has a similar distribution, with an $82 \mathrm{~km}$ long axis and a $15 \mathrm{~km}$ short axis. The area of $X$ level region is approximately $3144 \mathrm{~km}^{2}$, from southwest of Wenchuan County to northeast of Qingchuan County, with a $224 \mathrm{~km}$ long axis and a $28 \mathrm{~km}$ short axis. The shape of the IX level region is a long, narrow strip with an area of $7738 \mathrm{~km}^{2}$, and it has a $318 \mathrm{~km}$ long axis and a $45 \mathrm{~km}$ short axis. The VIII, VII, and VI level regions are similar, irregular ellipses with areas of $27,786 \mathrm{~km}^{2}, 84,449 \mathrm{~km}^{2}$, and $314,906 \mathrm{~km}^{2}$, respectively.

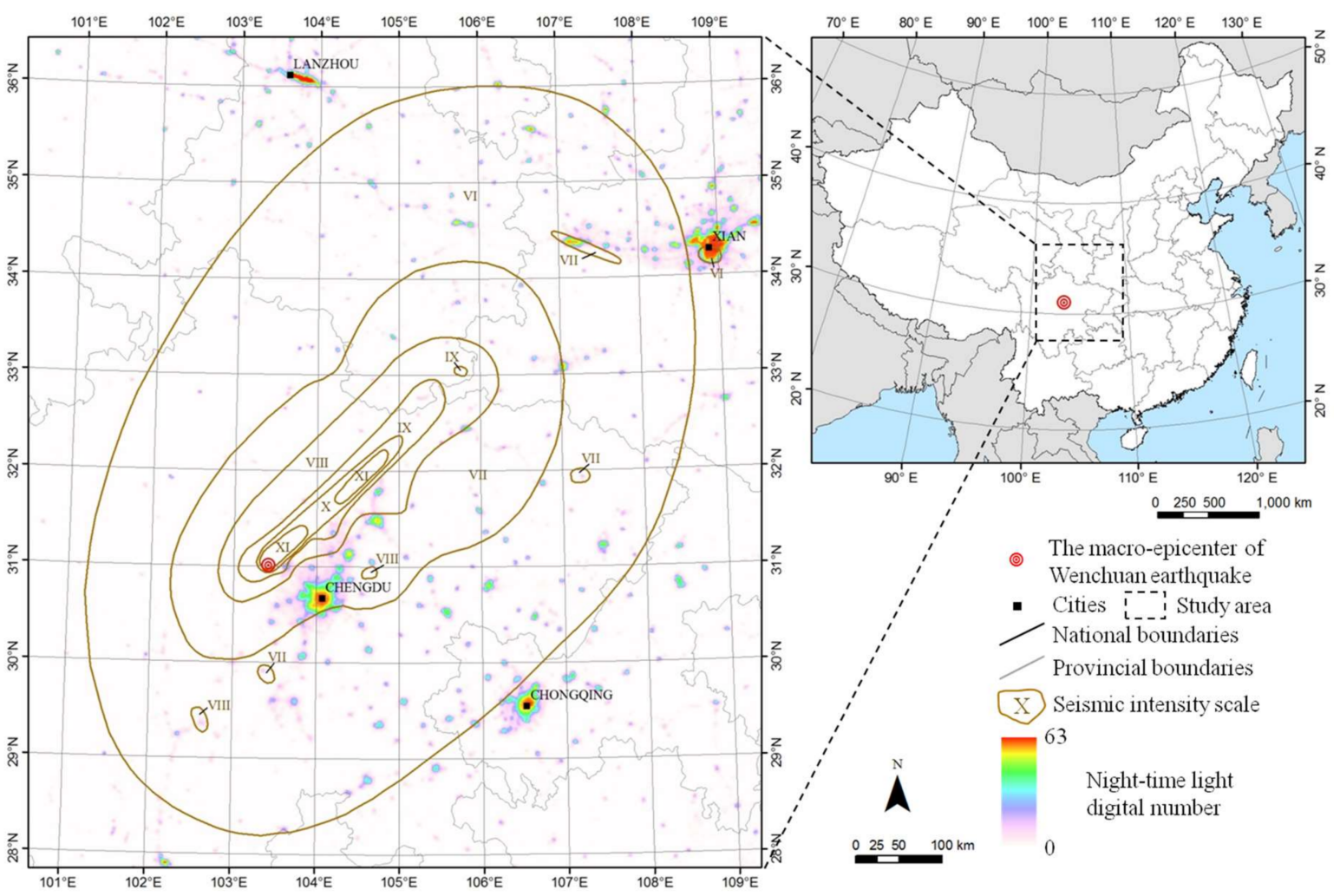

Figure 1. The study area of the research. The seismic intensity scale is the Chinese seismic intensity scale. The distribution of the Wenchuan earthquake seismic intensity comes from the field survey from the China Earthquake Administration. The NTL image used in the figure is obtained in 2008 by DMSP/OLS.

\subsection{Data and Preprocessing}

This study used DMSP/OLS NTL cloud-free annual composites images spanning over 11 years, from 2003 to 2013, from the V4DNLTS dataset. Data were obtained from the National Centers for Environment Information (NCEI, formerly NGDC) at the National Oceanic and Atmospheric Administration (NOAA) website [25] and included data acquired by three DMSP satellites: F15, F16, and F18 (Table 1). These data are recorded in two formats: frequency of lighting and average stable 
light. For this study, focused on the stable light product, which has a 6-b radiometric resolution such that the digital number (DN) values range from 0 (no lighting) to 63 (highest lighting), with a spatial resolution of 30 arc-seconds (about 1-km spatial resolution at the equator).

Table 1. Annual composites produced.

\begin{tabular}{llll}
\hline Year & & Satellite & \\
\hline $\mathbf{2 0 0 3}$ & F15 & & \\
$\mathbf{2 0 0 4}$ & F15 & F16 & \\
$\mathbf{2 0 0 5}$ & F15 & F16 & \\
$\mathbf{2 0 0 6}$ & F15 & F16 & \\
$\mathbf{2 0 0 7}$ & F15 & F16 & \\
$\mathbf{2 0 0 8}$ & & F16 & \\
$\mathbf{2 0 0 9}$ & & F16 & \\
$\mathbf{2 0 1 0}$ & & & F18 \\
$\mathbf{2 0 1 1}$ & & & F18 \\
$\mathbf{2 0 1 2}$ & & & F18 \\
$\mathbf{2 0 1 3}$ & & & F18 \\
\hline
\end{tabular}

The original DMSP/OLS time series NTL images are not strictly registered with each other (Figure 2). To reduce the influence caused by image registration error, the time series NTL images were uniformly reprojected onto the UTM-WGS84-49N coordinate system, and the pixel size was resampled to $1-\mathrm{km}$.

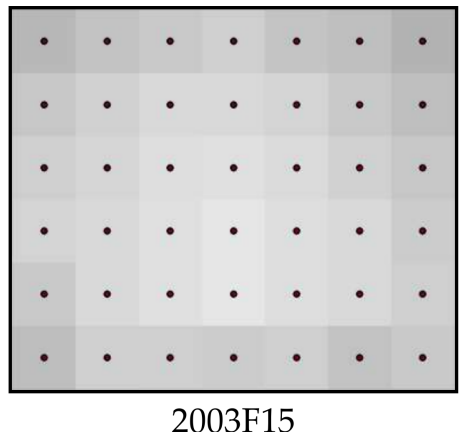

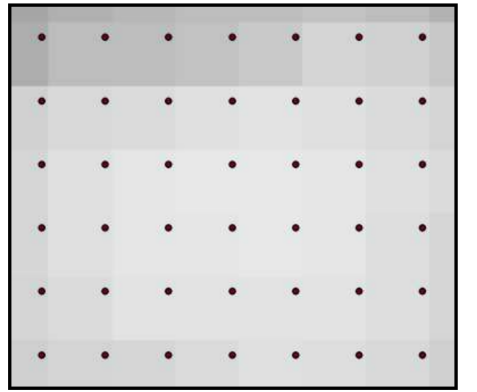

2009F16

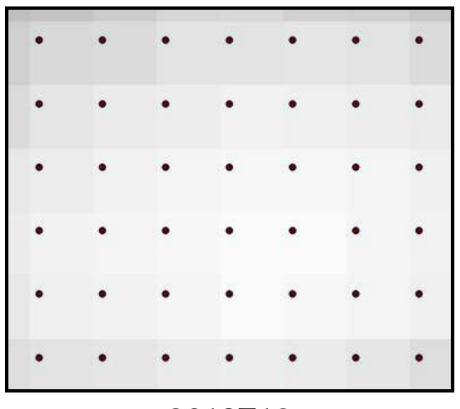

2013F18

Figure 2. The registration error of the DMSP/OLS time series NTL images in the same region. The $\operatorname{dot}(\mathrm{s})$ represents the pixel center of the reference image, which is defined as an NTL image acquired by the F16 satellite in 2008.

\subsection{Automatic Intercalibration Method}

As noted above, NTL data in the V4DNLTS dataset lack continuity and comparability and, thus, they cannot be used directly to extract the dynamics of HA in areas affected by the Wenchuan earthquake. Many scholars have proposed different intercalibration methods to correct the global or local NTL images. The most commonly used methods are shown in Table 2. Most of the existing calibration methods follow Elvidge's paradigm, but use different calibration sites [18,19,21]. In addition, Zhang and Li have used a globally-consistent and regionally-consistent bias, respectively, to calibrate the NTL images [22,24].

The goals of all NTL calibration methods are similar in principle. That is to create a consistent time series so that different images are directly comparable. The calibration method presented by Elvidge requires manual selection of the unchanged region. The quality of invariant regional selection has a great impact on calibration results. When dealing with a rapidly-developing local region, this dynamic becomes a challenge. Liu and Yu use the same intercalibration method as Elvidge's [19,21]. Although they all adopt a post-adjustment after intercalibration to improve the consistency of the time series NTL 
data, the defect that samples are selected manually is still not resolved. The precondition of Zhang's method can only hold at the global scale, since, at local scales, the majority of the pixels might not be treated as pseudoinvariant features, such as Beijing, China, which experiences a great deal of change over a relatively short period. Li assumed that on-ground changes appear as outliers in a scatterplot generated from two NTL image pairs and that a linear equation can correct systematic biases after removing the outliers; this represents an automatic approach eliminating the need to manually choose the stable regions. However, it is difficult to distinguish between on-ground changes and systematic biases only using two-image pairs, especially when the surface changes rapidly. Furthermore, the linear regression is not always optimal.

Table 2. Differences in the methodological details of existing intercalibration methods.

\begin{tabular}{cccccc}
\hline $\begin{array}{c}\text { Intercalibration } \\
\text { Methods }\end{array}$ & $\begin{array}{c}\text { Application } \\
\text { Region }\end{array}$ & $\begin{array}{c}\text { Calibration } \\
\text { Site }\end{array}$ & $\begin{array}{c}\text { Reference } \\
\text { Image }\end{array}$ & $\begin{array}{c}\text { Time } \\
\text { Series }\end{array}$ & Precondition \\
\hline Elvidge [18] & Global & Sicily & 1999F12 & 1994-2008 & $\begin{array}{c}\text { Find the region with stable } \\
\text { NTL value in time series. }\end{array}$ \\
\hline Liu [19] & $\begin{array}{c}\text { Regional: } \\
\text { China }\end{array}$ & $\begin{array}{c}\text { Jixi city in } \\
\text { Heilongjiang } \\
\text { Province }\end{array}$ & 2007F16 & 1992-2008 & $\begin{array}{c}\text { Find the region with stable } \\
\text { NTL value in time series. }\end{array}$ \\
\hline Yu [21] & $\begin{array}{c}\text { Regional: } \\
\text { China }\end{array}$ & Sicily & 2003F15 & 1992-2013 & $\begin{array}{c}\text { Find the region with stable } \\
\text { NTL value in time series. }\end{array}$ \\
\hline Zhang [22] & Global & $\begin{array}{c}\text { None } \\
\text { (used globally } \\
\text { consistent bias) }\end{array}$ & 2000F15 & 1992-2012 & $\begin{array}{c}\text { Changes over a relatively } \\
\text { short period between the } \\
\text { reference and target images } \\
\text { taken at different time } \\
\text { points are minimal. }\end{array}$ \\
\hline Li [24] & $\begin{array}{c}\text { Regional: } \\
\text { Beijing }\end{array}$ & $\begin{array}{c}\text { None (used } \\
\text { regionally } \\
\text { consistent bias) }\end{array}$ & 2001F15 & 1992-2010 & $\begin{array}{c}\text { There are enough pixels } \\
\text { with stable lights in the two } \\
\text { DMSP/OLS images. }\end{array}$ \\
\hline
\end{tabular}

After analyzing the above methods, it was assumed that the time-series features of NTL images should be used to minimize the effects of on-ground changes and that the artificial error in the selection of stable regions could be avoided. To overcome the shortcomings of the above methods, a novel automatic intercalibration method was proposed. The hypothesis was as follows:

If there are stable pixels on the time series NTL images, the change ranges of their NTL value should be less than other pixels. Stable pixels refer to the consistently lit pixels with little on-ground changes.

Based on the above assumptions, an automatic iteration process was used to search for the pixels with stable NTL. Then, the coefficients of intercalibration were calculated based on these pixels. The automatic intercalibration algorithm has the following steps (Figure 3):

1. Let $\left\{\mathrm{T}_{1}, \mathrm{~T}_{2}, \ldots, \mathrm{T}_{\mathrm{n}}\right\}$ denote the time series NTL images, which have the same size; the vector $P_{\mathrm{i}}$ denote the $D N$ value of $\left\{T_{1}, T_{2}, \ldots, T_{n}\right\}$, written as $P_{i}=\left\{D N_{i 1}, D N_{i 2}, \ldots, D N_{i n}\right\}$, where $D_{i 1}$ denotes the $i$ th pixel value in image $T_{1}$ and $n$ denotes number of time series NTL images.

2. Calculate the mean and standard deviation of $P_{i}$, written as $a=\left\{a_{1}, a_{2}, \ldots, a_{m}\right\}$ and $b=\left\{b_{1}, b_{2}, \ldots, b_{m}\right\}$, where $a_{i}$ denotes the mean value of $P_{i}, b_{i}$ denotes the standard deviation value of $P_{i}$ and $m$ denotes the total number of the pixels in image $T_{1}$. At first, all the pixels in time series NTL images are employed as the samples.

3. Then, a quadratic polynomial regression is used to fit the scatterplots of $a$ and $b$.

4. The predicted values marked as $b^{\prime}$ are derived by entering the sample $a$ and the fitting parameters. Calculate the difference between $\mathrm{b}$ and $\mathrm{b}^{\prime}$ as $\Delta \mathrm{b}=\mathrm{b}-\mathrm{b}^{\prime}$ and derive the standard deviation of this difference as $S_{\Delta b}$. Then, define the outliers as the samples $\left\{\left(a_{i}, b_{i}\right): \Delta b_{i}<-k S_{\Delta b}\right\}$, where $\Delta b_{i}$ is the 
$i$ th element in the vector variable $\Delta \mathrm{b}$ and $\mathrm{k}$ is set to 2 in this study. If there is no outlier from the above definition, go to step 6 .

5. Update the two groups of samples by removing the samples $\left\{\left(a_{\mathrm{i}}, \mathrm{b}_{\mathrm{i}}\right): \Delta \mathrm{b}_{\mathrm{i}}>\mathrm{kS} \mathrm{S}_{\Delta \mathrm{b}}\right\}$ from pixel samples $a$ and $b$, and go back to step 3 .

6. Select the pixels below the last quadratic polynomial curve as the invariant pixels. Fix a reference image and intercalibrate the time series NTL images.

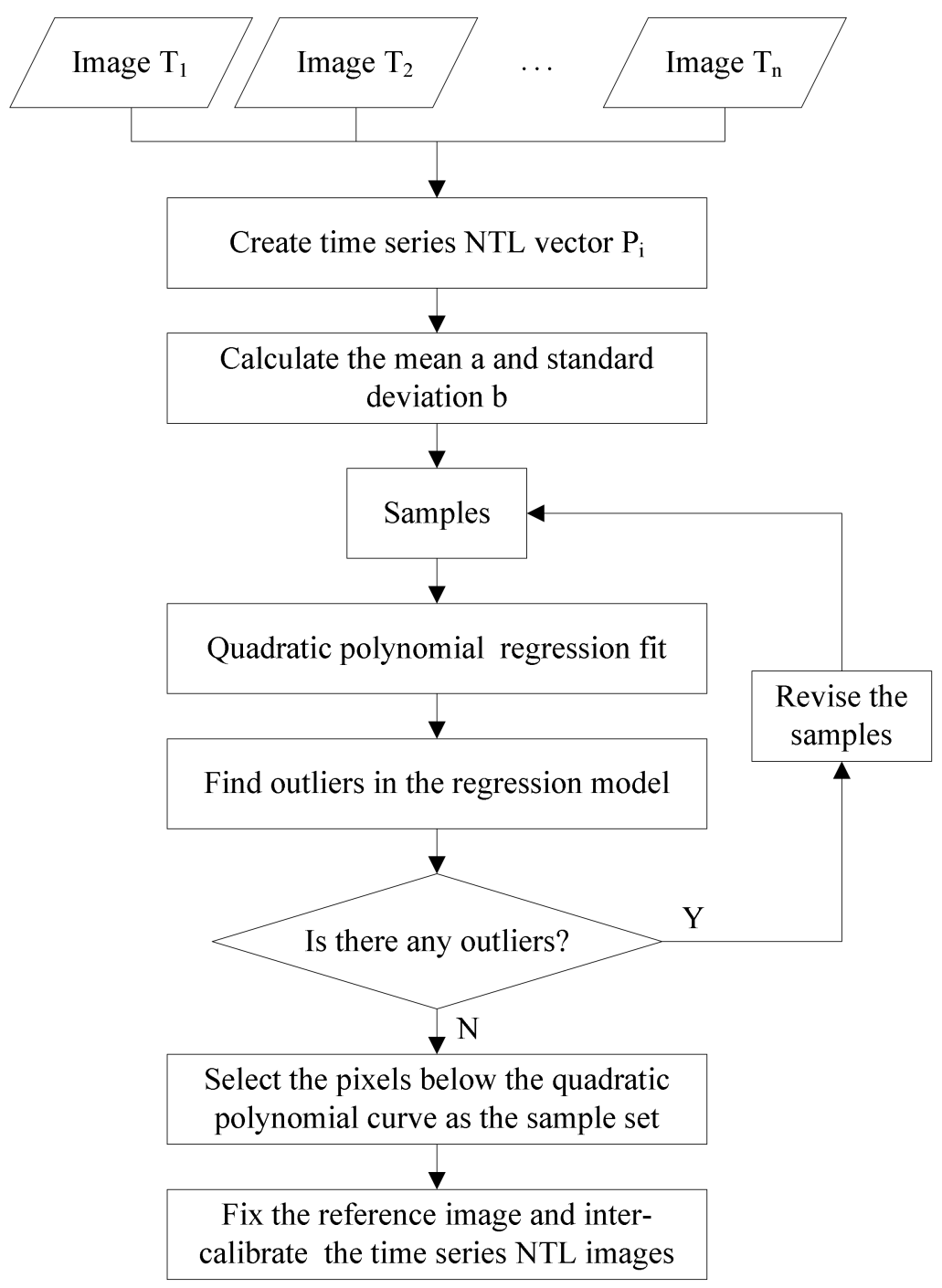

Figure 3. Flowchart of the proposed automatic intercalibration process.

\subsection{Classification of Human Activity Pattern}

The DN values of the NTL images are highly correlated with HA; this dynamic is independent and objective with spatiotemporal continuity. The calibrated NTL images were used to obtain the intensity of HA in the study area. To remove the intra-annual unstable lit pixels and to make full use of the information derived from the two satellites for the same year, an intra-annual composition was performed. First, we examined all lit pixels to identify whether the pixels were intra-annual unstable lit pixels. A lit pixel was defined as an intra-annual unstable lit pixel if it was detected by only one satellite. Second, in intra-annual composites, DN values of intra-annual unstable lit pixels 
were replaced with values of zero, and the DN value of each intra-annual stable lit pixel was replaced by the averaged DN value of two NTL images from the same year:

$$
\mathrm{DN}_{i n}=\left\{\begin{array}{lr}
0, & \mathrm{DN}_{\text {in }}^{F_{1}}=0 \mid \mathrm{DN}_{\text {in }}^{F_{2}}=0 \\
\left(\mathrm{DN}_{\text {in }}^{F_{1}}+\mathrm{DN}_{i n}^{F_{2}}\right) / 2, & \text { otherwise }
\end{array}\right.
$$

where $\mathrm{DN}_{i n}^{F_{1}}$ and $\mathrm{DN}_{i n}^{F_{2}}$ are DN values of the $i$ th lit pixel from two NTL images in the $n$th year, respectively, and $\mathrm{DN}_{i n}$ is the $\mathrm{DN}$ value of the $i$ th lit pixel of intra-annual composite in the $n$th year.

To facilitate the description of patterns of HA, HA are divided into these four levels: high HA, medium HA, low HA, and inactivity. High HA indicates that HA in the area is stable and well-lit, such as in cities. Medium HA indicates that HA in the area is stable, but less intense, such as in villages or settlements with smaller populations. Low HA indicates that HA in the area is temporary and unstable, such as during the construction of large projects.

Many methods are available to extract useful information from NTL imagery, including an empirical thresholding technique [26] and an image-classification method [27]. Of these methods, the thresholding technique with ancillary data has been widely used because of its simplicity and relatively high accuracy and reliability [28,29]. Therefore, we used the thresholding technique developed by Henderson [28], along with ancillary data, to extract the HA information in study area, after first systematically correcting NTL data for the 2003-2013 period. Notably, the long time interval will affect the consistency of time series NTL data. Although the intercalibration can correct the biases, a high level of inconsistencies remain at the pixel level in the calibrated NTL time series [22]. The ancillary data helps shorten the time intervals. This can improve the accuracy of subsequent analysis. Land cover data of International Geosphere-Biosphere Programme (IGBP) in 2005 and GLC30 data in 2010 were used as references to determine the thresholds of different HA levels and extracted the spatial extent of different HA levels during these two years (2005 and 2010) using land cover data (Table 3). These optimal thresholds were applied to other years in the time series; thus, the threshold for 2005 was applied to the period 2003-2008, and the threshold for 2010 was applied to the period 2009-2013.

In the study of urban extent extraction, a minimum DN value is usually set to exclude systematic biases, which often focus on low values. However, pixels with low DN value are one of the research objects which cannot be discarded in the study of HA. To balance the retention of useful information and bias interference, the lower limit of low HA was empirically defined as 1.

Table 3. Optimal thresholds of the human activity level in the study area for the years 2005 and 2010.

\begin{tabular}{ccccc}
\hline \multirow{2}{*}{$\begin{array}{c}\text { Human Activity } \\
\text { Level }\end{array}$} & \multicolumn{4}{c}{ Optimal Thresholds } \\
\cline { 2 - 5 } & 2005 Lower Limit & 2005 Upper Limit & 2010 Lower Limit & 2010 Upper Limit \\
\hline Inactivity & 0 & 1 & 0 & 1 \\
Low & 1 & 18 & 1 & 20 \\
Medium & 18 & 44 & 20 & 50 \\
High & 44 & 63 & 50 & 63 \\
\hline
\end{tabular}

\section{Results}

\subsection{Intercalibration}

After preprocessing, 15 DMSP/OLS NTL images from 2003 to 2013 were obtained with the same image size of $800 \times 920$ pixels, having lengths and widths of $1 \mathrm{~km}$. Notably, there are two images from different satellites every year between 2004 and 2007. A total of 619 invariant pixels were automatically selected as samples by the iterative filtration (Figure 4). These samples were considered to be stable from 2003 to 2013. Since the changes could be significant when the time interval between the reference image and the target image increases-and, furthermore, the quality of the F18 
images is lower than all other images [22]—we chose the 2008F16 image which lies in the middle of the DMSP/OLS time series as the reference image to minimize the effects from longer time intervals. Figure 5 shows the scattergrams for each of the target images versus the reference image for NTL samples. The second-order regression model developed for each image is shown in Table 4 . The adjusted DN was created through application of this formula:

$$
\mathrm{DN}_{\text {calibrated }}=a \times \mathrm{DN}^{2}+b \times \mathrm{DN}+c
$$

where $\mathrm{DN}$ is the original $\mathrm{DN}$ value, $\mathrm{DN}_{\text {calibrated }}$ is the intercalibrated $\mathrm{DN}$ value, and $a, b$, and $c$ are coefficients.

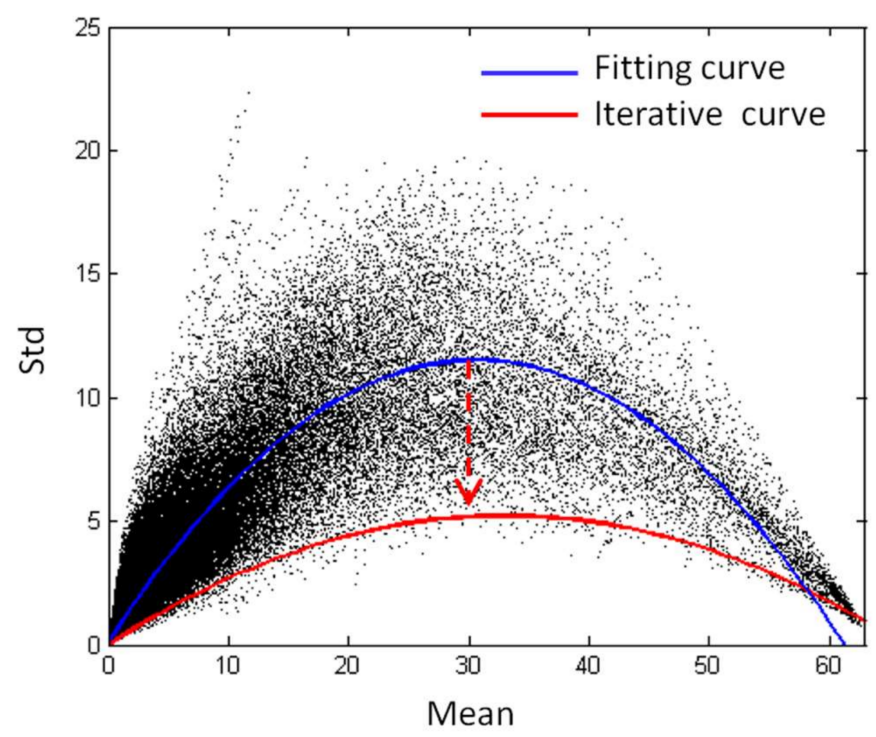

Figure 4. Scatter plot of mean and standard deviation of time series NTL images, where the dost denote the time series NTL pixel vector $P_{i}$, the fitting curve is fitted by all the pixels, and the iterative cure is calculated by the method described above. It takes three iterations to reach the final state. The dots, which are under the iterative curve, are selected as the invariant pixels.

Table 4. Coefficients of the second-order regression models for NTL data.

\begin{tabular}{ccccc}
\hline Images & $\boldsymbol{a}$ & $\boldsymbol{b}$ & $\boldsymbol{c}$ & $\mathbf{R} 2$ \\
\hline 2003F15 & -0.00224 & 1.15817 & 0.000258 & 0.995915 \\
2004F15 & 0.000074 & 0.967107 & 0.000339 & 0.996798 \\
$\mathbf{2 0 0 4 F 1 6}$ & 0.002351 & 0.827499 & 0.000238 & 0.99641 \\
2005F15 & -0.00272 & 1.174893 & -0.000009 & 0.997058 \\
2005F16 & -0.00037 & 1.009958 & 0.000263 & 0.997428 \\
2006F15 & -0.00138 & 1.083872 & 0.000015 & 0.997707 \\
2006F16 & 0.002208 & 0.835364 & 0.000292 & 0.996687 \\
2007F15 & -0.0024 & 1.159497 & 0.000252 & 0.997053 \\
2007F16 & 0.0016 & 0.899921 & 0.000051 & 0.998374 \\
2008F16 & 0 & 1 & 0 & 1 \\
2009F16 & -0.00084 & 1.053921 & 0.000435 & 0.995502 \\
2010F18 & 0.004577 & 0.683408 & 0.000079 & 0.998751 \\
2011F18 & 0.001055 & 0.907797 & 0.000191 & 0.997218 \\
2012F18 & 0.001431 & 0.872708 & 0.00017 & 0.997077 \\
2013F18 & 0.005026 & 0.630127 & 0.000544 & 0.98911 \\
\hline
\end{tabular}




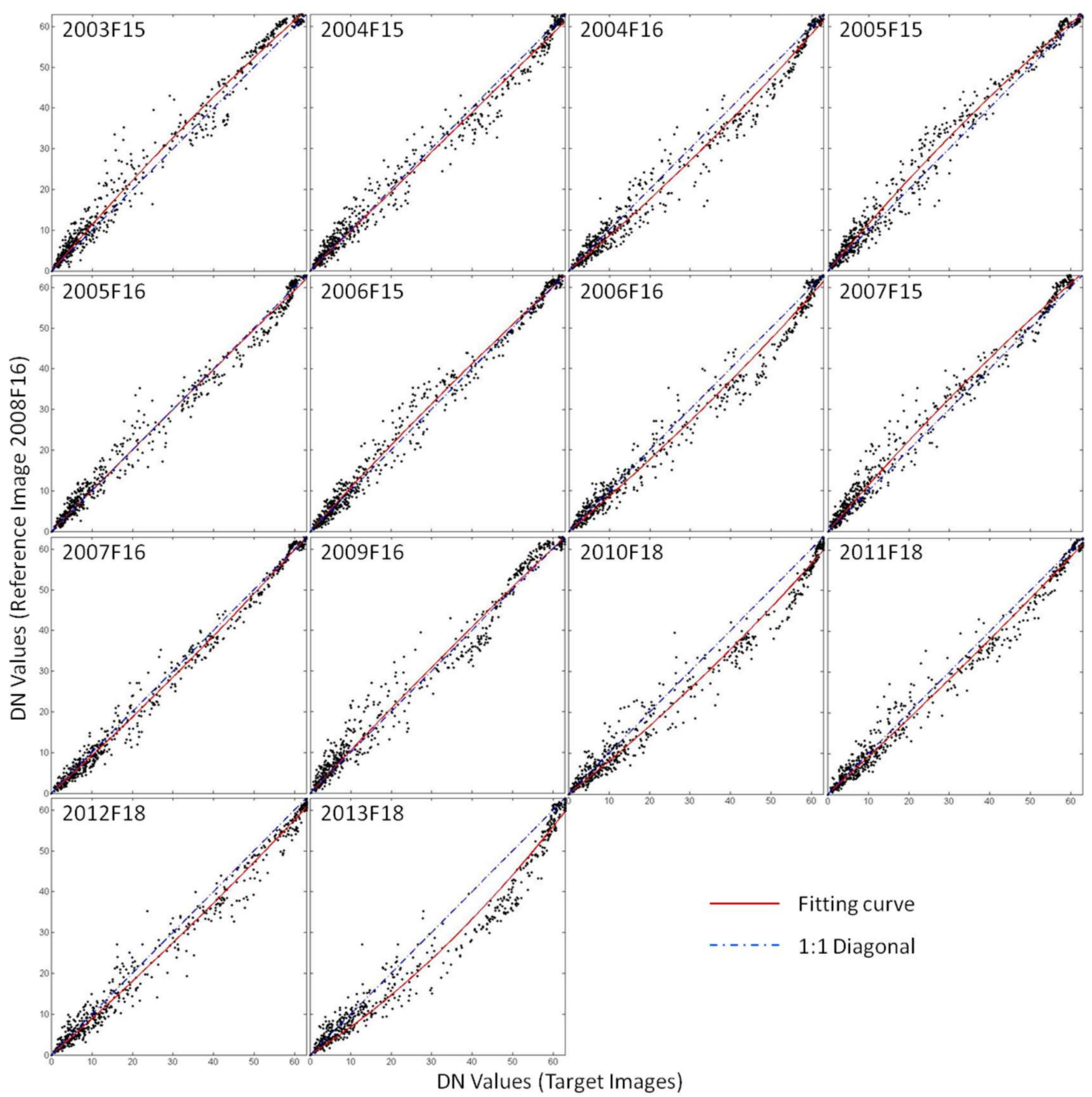

Figure 5. Scatter plots of each target image versus the reference image. The intercalibration was based on 619 lit pixels, which were selected iteratively by twice the standard deviation.

The total sum of NTL in the study area from both uncalibrated and calibrated DMSP-OLS images for the 11 years is illustrated graphically in Figure 6. The total sum of NTL in the calibrated image increases smoothly compared with that in the uncalibrated image, and the differences between two images in same year become smaller after calibration. This shows that the calibrated images are qualitatively better than that of the uncalibrated images.
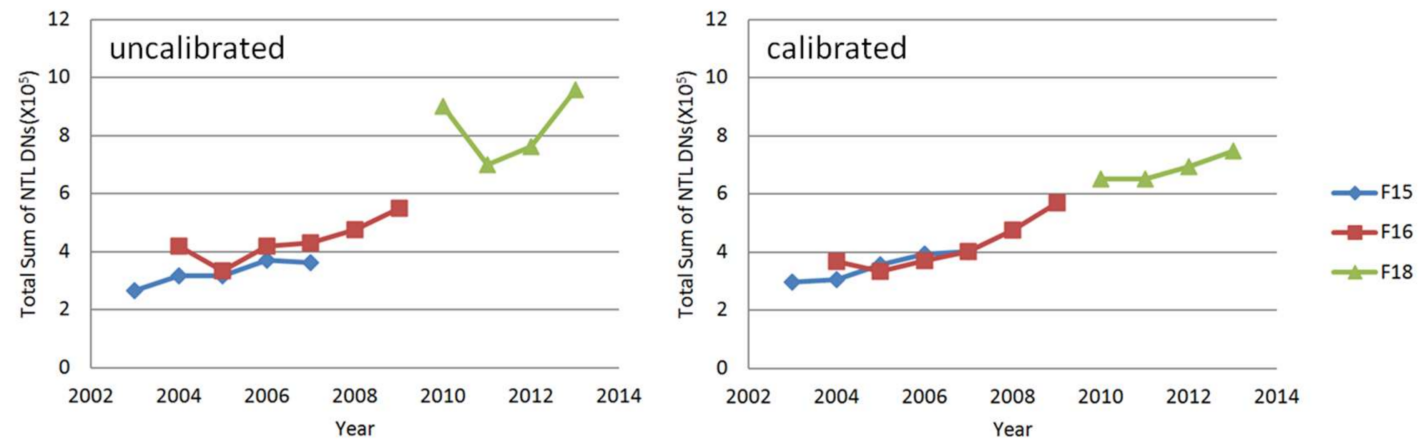

Figure 6. Total sum of NTL in the uncalibrated and calibrated DMSP-OLS images in the study area. 


\subsection{Human Activity Intensity Statistics}

Due to the high correlation to HA, DN of NTL image is used as a proxy to analyze the HA intensity changes. The total sum of NTL in different seismic intensity regions are counted. Since the size of each region differ, it is not meaningful to compare the quantity of HA intensity with each other. However, the changing trends of HA intensity in each seismic intensity region can be determined from the statistics. In order to make the changing trends comparable, the sum of NTL in each seismic intensity region is normalized from 0 to 1 (Figure 7).
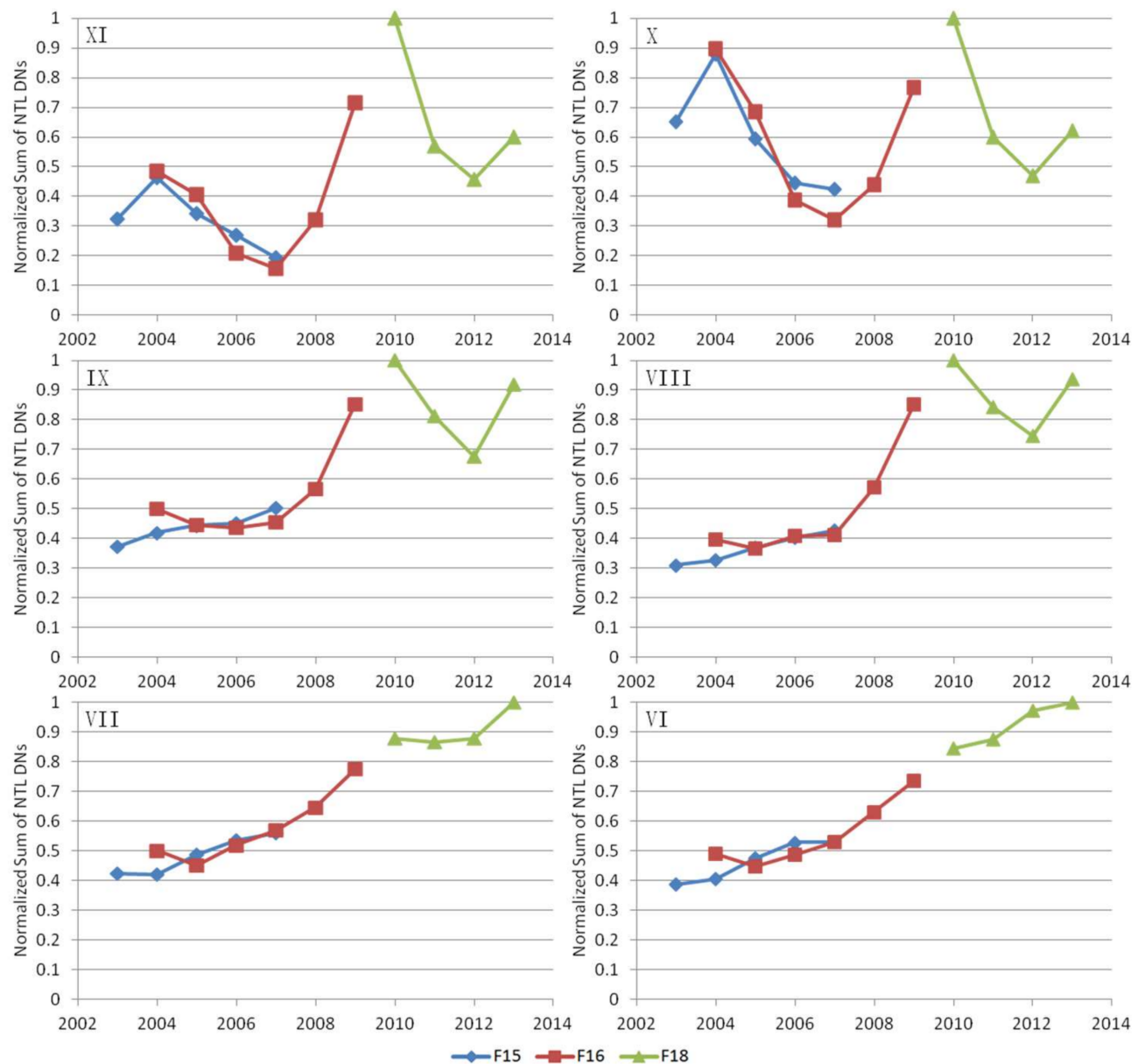

Figure 7. The changing trends in normalized sum of NTL in regions with different seismic intensities.

Seen from Figure 7, three kinds of changing trends can be identified roughly. The changes in the XI and X regions are similar. There are two peaks in 2004 and 2010 respectively, and the smallest appears in 2007. The slight difference is that the growth rate changing trend in the XI region from 2008 to 2010 is larger than that in the X region; the decreased value in the normalized sum of NTL in the XI region after 2010 is still higher than the peak in 2004, while the decreased value of the normalized sum of NTL in the X region after 2010 is much lower than the peak in 2004, only higher than the trough between 2006 and 2008.

There is consistency between the changes in the IX and VIII regions. The normalized sum of NTL between 2003 and 2007 is relatively stable. From the beginning of 2008, the total sum of NTL rose sharply and then started to fall after reaching its peak in 2010. However, the value after the fall is still higher than the value before 2008.

The normalized sum of NTL in the VII and VI regions tended to increase slowly over time. In the VII region, the acceleration increased slightly from 2008 to 2010 and then returned to normal with 
a slight slowdown between 2010 and 2012, while in the VI region the acceleration has always been relatively stable.

\subsection{Changes in Human Activity Levels}

To measure the changes in HA, we classified the NTL images into four levels based on the set of thresholds: high HA, medium HA, low HA, and inactivity. Figure 8 shows the spatiotemporal changes of HA levels in seriously-damaged regions. The spatiotemporal change of high HA reflects urban expansion in Chengdu. It seems that the expansion has not been affected by the Wenchuan earthquake, as do the changes of medium HA, which are mainly distributed in the VIII, VII, and VI regions.

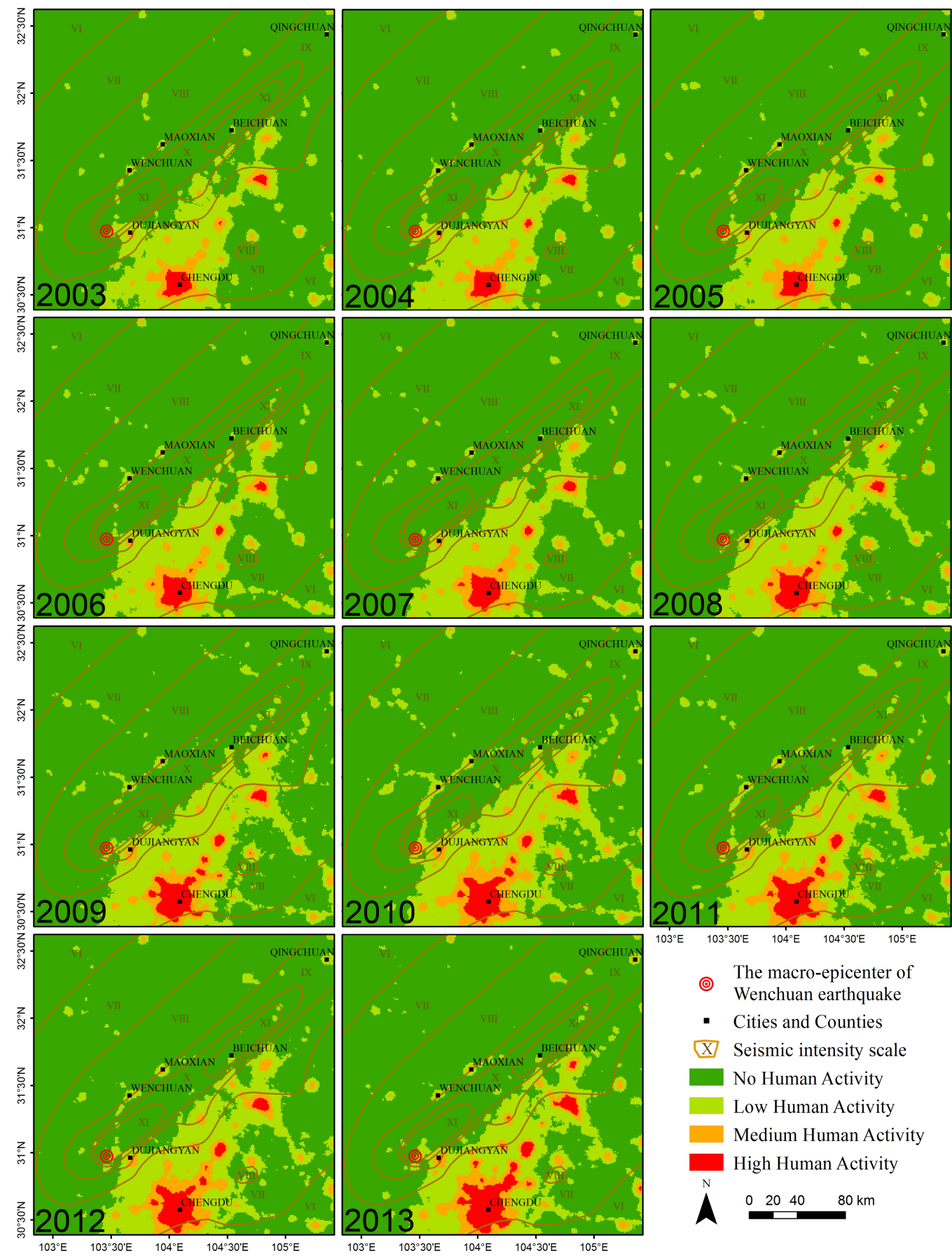

Figure 8. Time series changes of HA levels in seriously-damaged regions. 
Low HA is widely distributed. The spatiotemporal changes of low HA are more complicated. From 2003 to 2006, a strip of low HA from Dujiangyan to Wenchuan County were observed. This phenomenon disappeared in 2007 and reappeared in 2010, continuing until 2012. The banding of low HA passes through XI and X regions. The time of appearance and disappearance of the band coincided with the peak and low point in Figure 7. The recurrence of the banding of low HA from Dujiangyan to Wenchuan County coincided with the construction of Du-Wen highway, which began in 2003 and was completed in 2012. Similarly, the banding of low HA from Wenchuan County to Mao County which occurred in 2010 was also consistent with the restoration of State Road 213. So, too, does the band of low HA from Beichuan County to Qinchuan County in 2009 and 2010. These observations suggest that low HA may reflect the processes of large construction projects. In addition, HA of several counties in the severely-damaged regions (XI, X, and IX) saw gradual growth after 2008, such as Mao County and Wenchuan County. For the first time, medium HA in the middle of Mao County and Wenchuan County appeared in 2008 and in 2010, respectively, and expanded gradually. This demonstrates that post-earthquake recovery and reconstruction in the affected regions has led to local infrastructure and economic development.

\section{Discussion}

\subsection{Trends of Human Activity}

In Figure 7, the peaks in 2010 are observed in XI, X, IX, and VIII regions, which is similar with the uncalibrated NTL data in Figure 6. This phenomenon may be caused by two reasons. First, there are residual biases in the calibrated NTL data. Studies have shown that the intercalibration can correct the systematic biases, but it can be difficult to correct random noises [19,20]. Moreover, random noises mainly affect low NTL values. It will affect the sum of NTL when the statistical region is small and lacks enough well-lit pixels. Second, reconstruction after the Wenchuan earthquake led to development in these regions. Since the peaks in XI and X regions in 2004 are different from the uncalibrated NTL data and the areas of $\mathrm{XI}$ and $\mathrm{X}$ regions are small, the second reason is considered as the main factor. The increase in HA from 2008 to 2010 came from the implementation of various infrastructure projects, such as homes, roads, and bridges. Since 2011, major reconstruction and restoration projects have been completed in these regions, and development has gradually stabilized. Time series changes of HA levels shown in Figure 8 illustrate that the fluctuation of HA in XI and X regions before 2008 is consistent with the construction stage of the DU-Wen highway. For the VII and VI regions, HA changes caused by the Wenchuan earthquake are not obvious. This observation demonstrates that the Wenchuan earthquake had little impact on economic development in this area and that the rate of development in the VII and VI regions remained stable.

Notably, although the changing trends in the normalized sum of NTL in the XI and X regions before and after the Wenchuan earthquake are consistent, the proportion of the two changed after the earthquake. As seen in Figure 9, from 2003 to 2007, the ratio of the total sum of NTL in the XI and X regions remained between 0.8 and 1.0, while the total sum of NTL in the XI region never exceeded the quantity in the $X$ region before 2008. After the Wenchuan earthquake, the total sum of NTL in the XI region exceeded that in the $\mathrm{X}$ region for the first time. The proportion increased annually and stabilized at 1.6 after 2010. This suggests that patterns of HA in the XI and X regions were greatly changed by the reconstruction and recovery. The rise ratio of the total sum of NTL in the XI and X regions can be explained thusly: the damage in the XI region was more serious than the damage in the $\mathrm{X}$ region, and the $\mathrm{XI}$ region received more reconstruction and restoration attention, which led to greater HA in the XI region. This change not only occurred during the reconstruction period, but continued during the stabilization period. It seems that the earthquake destroyed this area, but the reconstruction made it better than before. We conclude that, with the post-earthquake reconstruction, the social and economic environment in the XI region has been restored and, indeed, surpassed the prior benchmark, which is the main reason for its rise in HA as compared to the $\mathrm{X}$ region. 


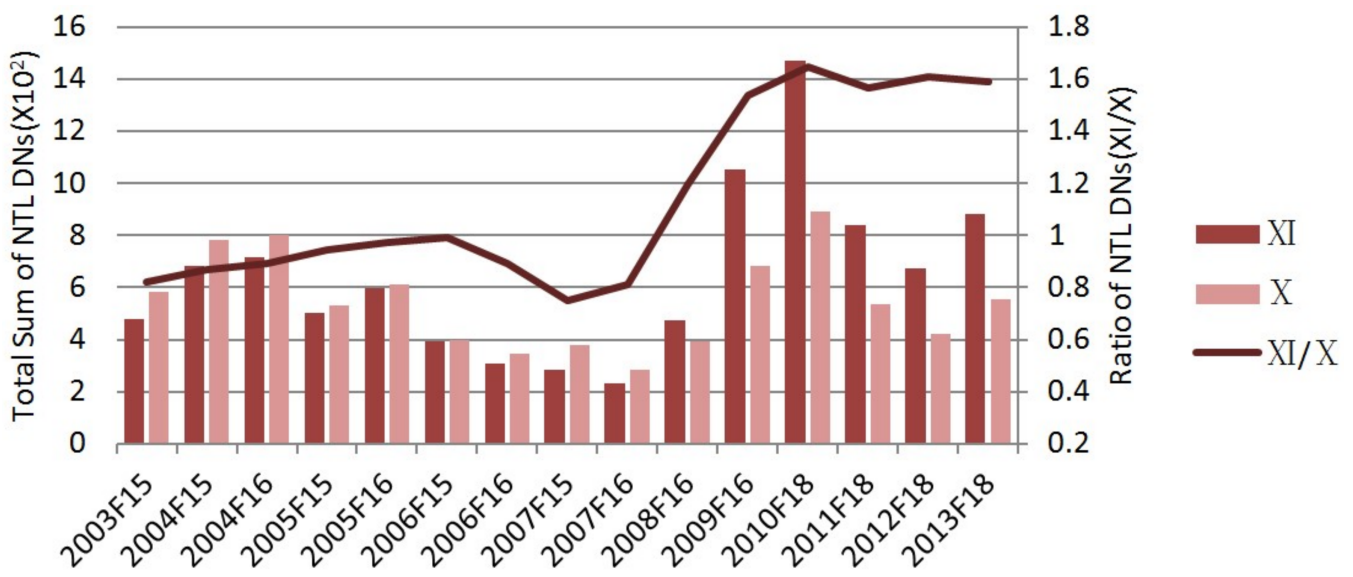

Figure 9. The change contrast in the total sum of NTL in the XI and X regions.

\subsection{Spatiotemporal Patterns of Human Activity}

To measure changes in patterns of HA, we created a statistic of the total number of patches (NP) and the total class area (CA) of a particular HA level (Figure 10). Obviously, after the Wenchuan Earthquake, the patterns of low HA in different seismic intensity regions changed. In the XI region, there was a pattern of increase, first, and then a decrease before 2008; following this there was a remarkable increase after 2008 and a decrease after 2010. However, in the X region, the pattern of low HA was relatively stable before 2008; after a slight increase from 2008 to 2010, there was a drop to a lower, pre-earthquake level. The pattern change of low HA in other seismic intensity regions were similar; that is, stable before the earthquake and showing a different magnitude of increase between 2008 and 2010, followed by a steady or slight decline. It is noted that, in some years, the increased ratio of NP was larger than that of CA. This indicates that the low HA in these regions were more dispersed in these years.

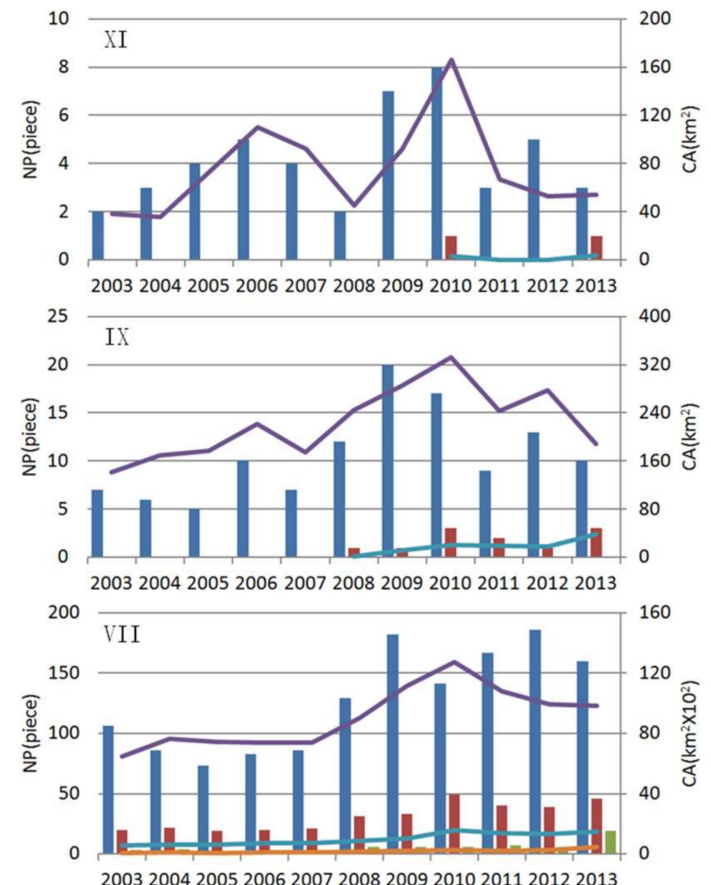

Year
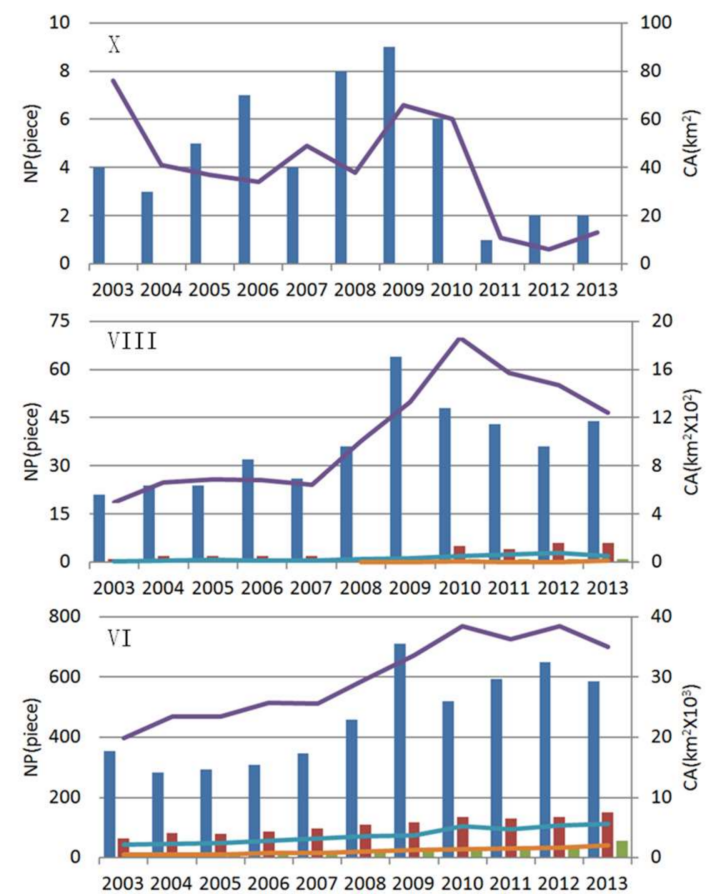

NP_MediumHA NP_HighHA Year

-CA_LowHA CA_MediumHA C CA_HighHA

Figure 10. The statistic of human activity spatiotemporal pattern in the study area. 
Medium HA was continuous in VIII, VII, and VI regions from 2003 to 2013. It tended to show a slow increase. Moreover, the increased rates between 2009 and 2010 were slightly larger than in other years. In the IX region, the stable medium HA appeared after 2008, while there was no stable medium HA appeared before and after the earthquake in XI and X regions. The spatiotemporal distribution of high HA is similar to medium HA, but the variation of high HA is smaller.

In conclusion, the reconstruction and recovery of the Wenchuan earthquake affected the spatiotemporal distribution of low HA in each seismic intensity region. Low HA in most regions after reconstruction are more active than before 2008, except that in the $\mathrm{X}$ region. This may be caused by the lack of stable settlements in the $X$ region. Medium HA seems to be less affected by the earthquake and recovery, while the changes in high HA do not seem to be affected by the restoration of the Wenchuan earthquake.

\subsection{Future Research}

The intercalibration method for DMSP/OLS images is the basis of this study. The automatic intercalibration method proposed in this paper can be used not only for the assessment of the impact of natural disasters, but also for the spatial and temporal dynamics of settlement, demographics, and socioeconomic parameters. Although the automatic intercalibration method successfully calibrates the DMSP/OLS time series NTL images of the Wenchuan earthquake, it still has two main limitations. First, the method is only applied locally. It will face more challenges when applied in global scale. Second, the assumption that there are stable pixels on the time series NTL images can be at risk when the time interval of time series get larger. In addition, although the annual NTL data were used to study the long-term impacts of the Wenchuan earthquake, monthly and daily NTL data are considered to be able to show more details of reconstruction and recovery. Finally, the DN value of NTL images is only an approximation of HA. Anomalies in the NTL, such as ice reflection, cloud reflection, and natural flame, can affect the results of HA analysis. The lack of effective methods for extracting HA from NTL images limits further research. All above problems need to be studied and solved in the future.

\section{Conclusions}

The long-term impact of earthquakes' destruction and restoration is a pivotal issue. In this paper, time series DMSP/OLS NTL images were used to evaluate changes in HA in Wenchuan earthquake-stricken areas, from 2003 to 2013. To improve the continuity and comparability of NTL data in the V4DNLTS dataset, this study developed an automatic intercalibration method to systematically correct NTL data. Based on the calibrated NTL images, the changes of intensity and the spatiotemporal pattern of HA in different seismic intensity regions were analyzed.

The results revealed that the sum of NTL in the XI and X regions fluctuated before and after the Wenchuan earthquake. While, in the IX and VIII regions, the sum of NTL remained stable before 2008 and began to rise from 2008 to 2010, then fell after 2010. However, the sum of NTL in the IX and VIII regions after 2010 was still higher than before 2008. The sum of NTL in the VII and VI regions maintained a steady growth, and did not show an obvious impact from the earthquake. Comparing the sum of NTL in the XI and X regions, we found that, before 2008, the sum of NTL in the XI region was always less than that in the X region, but after 2008, the ratio of the sum of NTL in these two regions increased rapidly and stabilized at 1.6 after 2010. This finding illustrated that the post-earthquake reconstruction efforts in the $\mathrm{XI}$ region should have been far greater than in the $\mathrm{X}$ region.

A classification of HA was used to analyze the pattern changes in different seismic intensity regions. The study found that the low HA in the vicinity of the Wenchuan earthquake epicenter was closely related to the construction of roads, such as the Du-Wen Highway and the 213 State Road, which also explained the fluctuation in the sum of NTL in the XI and X regions. We also found the correlation between post-earthquake reconstruction and changes in HA, with low HA in different seismic intensity regions significantly increased from 2008 to 2010. Medium HA in the VIII, VII, and VI 
regions slightly increased since 2008, and the high HA maintained steady growth and, thus, were not notably impacted by the reconstruction and recovery projects of the Wenchuan earthquake.

Acknowledgments: This research was funded by foundation of director of Institute of Seismology, CEA (Grant No. IS201616248) and the National Natural Science Foundation of China (Grant No. 41401428). The authors acknowledge Li Xi from Wuhan University, Wuhan, China, for suggestions on improving the intercalibration methods.

Author Contributions: For this article, Xue Li and Cong Zhan analysed the data and performed the experiments; Liang Li collected and processed the survey data of Wenchuan earthquake and land cover data from IGBP and GLC30; Xue Li wrote the paper; and Jianbing Tao contributed to the design of the research, article structure, and the acquisition of the DMSP/OLS imagery. All authors contributed to the editing of the article.

Conflicts of Interest: The authors declare no conflict of interest.

\section{Appendix A}

The Chinese Seismic Intensity (CSI) scale is similar with Modified Mercalli Intensity (MMI) scale, which is used by the United States Geological Survey (USGS). Both of CSI and MMI have 12 degrees (from I-not felt-to XII-total destruction) to quantify the effects of an earthquake on the Earth's surface, humans, objects of nature, and man-made structures. However, because of the slight differences in the descriptions of 12 levels of CSI and MMI, they are not completely consistent. Fortunately, the link between CSI and MMI can be inferred with the ground motion parameters, such as peak acceleration and peak velocity (Figure A1, Table A1).

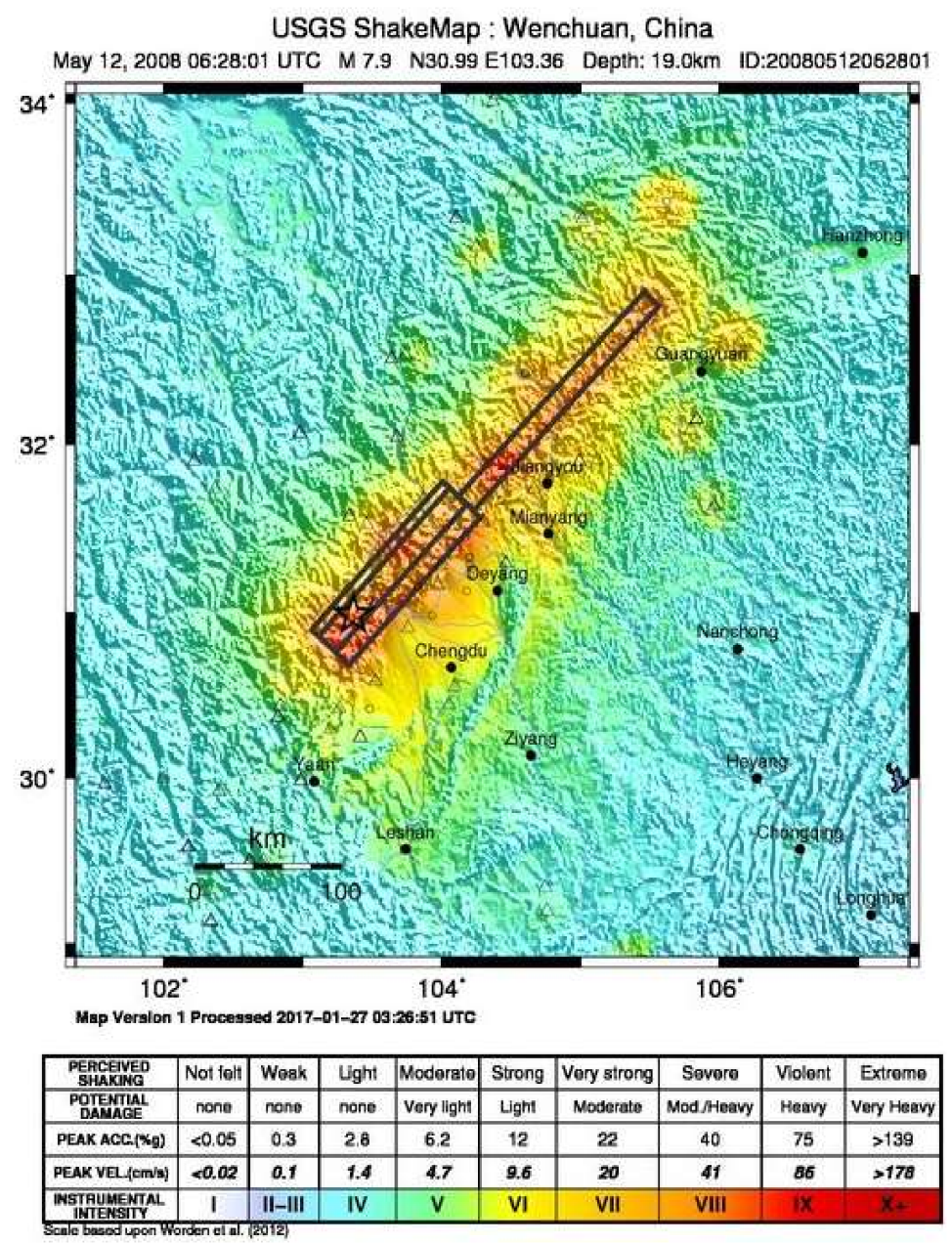

Figure A1. The USGS ShakeMap of the 2008 Wenchuan, China earthquake. 
Table A1. The abbreviated Chinese seismic intensity scale.

\begin{tabular}{ccccccccc}
\hline Chinese Seismic Intensity & I-IV & V & VI & VII & VIII & IX & X & XI+ \\
\hline Peak ACC $(\% g)$ & $<2.2$ & $2.2-4.5$ & $4.6-9.1$ & $9.2-18.1$ & $18.2-36$ & $36.1-72.1$ & $72.2-144.3$ & $>144.3$ \\
Peak VEL $(\mathrm{cm} / \mathrm{s})$ & $<2$ & $2-4$ & $5-9$ & $10-18$ & $19-35$ & $36-71$ & $72-141$ & $>141$ \\
\hline
\end{tabular}

\section{References}

1. Statistics on Casualties and Economic Losses of Wenchuan Earthquake on 12 May 2008. Available online: https:/ /baike.baidu.com/item/5\%C2\%B712\%E6\%B1\%B6\%E5\%B7\%9D\%E5\%9C\%B0\%E9\%9C\%87/ 11042644\#32 (accessed on 1 September 2017).

2. Li, D.; Chen, X.; Cai, X. Spatial information techniques in rapid response to Wenchuan Earthquake. J. Remote Sens. 2008, 12, 841-851.

3. Wei, C.; Liu, Y.; Wang, S.; Zhang, L.; Huang, X. Investigation and assessment of damage in earthquake Wenchuan Sichuan quake based on remote sensing. J. Remote Sens. 2008, 12, 673-682.

4. Zeng, T.; Yang, W.; Li, X.; Liu, H.; Peng, L.; Jian, J. Information extraction of object-oriented high resolution remote sensing image: A case study on urban damaged buildings in Wenchuan Earthquake. J. Nat. Disasters 2010, 19, 81-87. [CrossRef]

5. Wang, X.; Wang, L.; Zhang, X.; Ding, X.; Qiu, H.; Dong, Y.; Dou, A.; Zhang, F.; Liu, Z. Primary quantitative study on earthquake damage extracted from remote sensing imagery: A case study of Douj iangyan due to the Wenchuan M8.0 earthquake. Earthquake 2009, 29, 174-181.

6. Xu, C.; Dai, F.; Xu, X. Wenchuan Earthquake-induced landslides: An overview. Geol. Rev. 2010, 56, 860-874. [CrossRef]

7. Cui, P.; Lin, Y.; Chen, C. Destruction of vegetation due to geo-hazards and its environmental impacts in the Wenchuan Earthquake areas. Ecol. Eng. 2012, 44, 61-69. [CrossRef]

8. Fan, J.; Zhang, J.; Tian, B.; Yan, D.; Tao, H. Investigation to the damaged farmland as a result of disasters induced by Wenchuan Earthquake based on remote sensing: A case study of Tangjiashan area, Beichuan County, Sichuan Province. J. Remote Sens. 2008, 12, 917-924.

9. Di, B.; Zeng, H.; Zhang, M.; Ustin, S.L.; Tang, Y.; Wang, Z.; Chen, N.; Zhang, B. Quantifying the spatial distribution of soil mass wasting processes after the 2008 earthquake in Wenchuan, China. A case study of the Longmenshan area. Remote Sens. Environ. 2010, 114, 761-771. [CrossRef]

10. Xu, X.; Jiang, D.; Zhuang, D.; Qiu, D. Assessment about the impact of Wenchuan Earthquake on ecological environment. Acta Ecol. Sin. 2008, 28, 5899-5908. [CrossRef]

11. Huang, R.; Li, W. Post-earthquake landsliding and long-term impacts in the Wenchuan Earthquake area, China. Eng. Geol. 2014, 182, 111-120. [CrossRef]

12. Zhang, S.; Zhang, L.M. Impact of the 2008 Wenchuan Earthquake in China on subsequent long-term debris flow activities in the epicentral area. Geomorphology 2017, 276, 86-103. [CrossRef]

13. Li, D.; Li, X. An overview on data mining of nighttime light remote sensing. Acta Geod. Cartogr. Sin. 2015, 44, 591-601. [CrossRef]

14. Li, X.; Zhang, R.; Huang, C.; Li, D. Detecting 2014 Northern Iraq Insurgency using night-time light imagery. Int. J. Remote Sens. 2015, 36, 3446-3458. [CrossRef]

15. Kohiyama, M.; Hayashi, H.; Maki, N.; Higashida, M.; Kroehl, H.W.; Elvidge, C.D.; Hobson, V.R. Early damaged area estimation system using DMSP-OLS night-time imagery. Int. J. Remote Sens. 2004, 25, 2015-2036. [CrossRef]

16. Koma, H.; Akatsuka, S.; Sawada, H. DMSP-OLS for observing the reconstruction stage of the damaged area caused by the Sichuan earthquake. In Proceedings of the 30th Asian Conference Remote Sensing, Beijing, China, 18-23 October 2009.

17. Gillespie, T.W.; Frankenberg, E.; Chum, K.A.I.F. Nighttime lights time series of tsunami damage, recovery, and economic metrics in Sumatra, Indonesia. NIH Public Access 2015, 5, 286-294. [CrossRef]

18. Elvidge, C.D.; Ziskin, D.; Baugh, K.E.; Tuttle, B.T.; Ghosh, T.; Pack, D.W.; Erwin, E.H.; Zhizhin, M. A fifteen year record of global natural gas flaring derived from satellite data. Energies 2009, 2, 595-622. [CrossRef]

19. Liu, Z.; He, C.; Zhang, Q.; Huang, Q.; Yang, Y. Extracting the dynamics of urban expansion in China using DMSP-OLS nighttime light data from 1992 to 2008. Landsc. Urban Plan. 2012, 106, 62-72. [CrossRef] 
20. Hsu, F.C.; Baugh, K.E.; Ghosh, T.; Zhizhin, M.; Elvidge, C.D. DMSP-OLS radiance calibrated nighttime lights time series with intercalibration. Remote Sens. 2015, 7, 1855-1876. [CrossRef]

21. Yu, S.; Zhang, Z.; Liu, F. Monitoring Population Evolution in China Using Time-Series DMSP/OLS Nightlight Imagery. Remote Sens. 2018, 10, 194. [CrossRef]

22. Zhang, Q.; Pandey, B.; Seto, K.C. A Robust Method to Generate a Consistent Time Series From DMSP/OLS Nighttime Light Data. IEEE Trans. Geosci. Remote Sens. 2016, 54, 1-11. [CrossRef]

23. Pandey, B.; Zhang, Q.; Seto, K.C. Comparative evaluation of relative calibration methods for DMSP/OLS nighttime lights. Remote Sens. Environ. 2017, 195, 67-78. [CrossRef]

24. Li, X.; Chen, X.; Zhao, Y.; Xu, J.; Chen, F.; Li, H. Automatic intercalibration of night-time light imagery using robust regression. Remote Sens. Lett. 2013, 4, 46-55. [CrossRef]

25. Version 4 DMSP-OLS Nighttime Lights Time Series. Available online: https://www.ngdc.noaa.gov/eog/ dmsp/downloadV4composites.html (accessed on 20 November 2017).

26. Elvidge, C.D.; Baugh, K.E.; Kihn, E.A.; Kroehl, H.W.; Davis, E.R. Mapping city lights with nighttime data from the DMSP Operational Linescan System. Photogramm. Eng. Remote Sens. 1997, 63, 727-734.

27. Cao, X.; Chen, J.; Imura, H.; Higashi, O. A SVM-based method to extract urban areas from DMSP-OLS and SPOT VGT data. Remote Sens. Environ. 2009, 113, 2205-2209. [CrossRef]

28. Henderson, M.; Yeh, E.T.; Gong, P.; Elvidge, C.D.; Baugh, K.E. Validation of urban boundaries derived from global night-time satellite imagery. Int. J. Remote Sens. 2003, 24, 595-609. [CrossRef]

29. Milesi, C.; Elvidge, C.D.; Nemani, R.R.; Running, S.W. Assessing the impact of urban land development on net primary productivity in the southeastern United States. Remote Sens. Environ. 2003, 86, 401-410. [CrossRef]

(C) 2018 by the authors. Licensee MDPI, Basel, Switzerland. This article is an open access article distributed under the terms and conditions of the Creative Commons Attribution (CC BY) license (http:/ / creativecommons.org/licenses/by/4.0/). 